The two-qubit gate implemented by the authors can be seen as a CNOT gate, in which qubit information is encoded in quantum 'phases. To picture what this means, imagine an arbitrary state that can be represented by $x, y$ and $z$ axes, positioned at the north pole of a sphere (Fig. 1a). Simply rotating the system around its $z$ axis produces a gain of phase: a change in the orientation of the $x$ and $y$ axes, which can be represented by the angle of rotation of the $x$ axis within the $x-y$ plane.

However, to implement a two-qubit gate, the phase of the gate must depend on which states are adopted by the control and target qubits, and thus must be acquired in a fundamentally different way. If the system of axes is moved down the surface of the sphere to the equator, then along the equator for a short distance and back up to the north pole, the net result is again a rotation of the $x$ axis (Fig. 1b). This phase change is proportional to the area enclosed by the pathway taken, and is referred to as geometric phase.

Returning to Schäfer and colleagues' work, the motion of a pair of ions along the pair's axis can be described in terms of two vibrational modes - one in which the ions oscillate in sync with each other, and the second in which they oscillate in opposite directions. Oscillation in these modes correlates the ions' positions and momenta, thus defining a 'phase space' for their collective motion and for geometric phase generation, analogous to the surface of the sphere mentioned above. The motion of the ions can be controlled using lasers, which induce a shift of the ions' electronic states that depends on the position of the ions. Position-dependent shifts of states also generate a force. Because the qubit of a trapped ion is encoded by electronic states, the force exerted on the ions by the lasers depends on the state of the qubit.

If two overlapped, coherent laser beams (that is, laser beams whose light waves are in sync) are used, they produce a standing wave. If the frequency of one of the lasers is tuned slightly away from the frequency of the other (corresponding to a frequency change of $\delta$ ), then the standing wave starts to move. If $\delta$ is the same as the resonance frequency of one of the ions' vibrational modes, then the wave shakes the ions.

However, to induce geometric phase changes, $\delta$ must not be at the resonance frequency of the modes. At off-resonance frequencies of $\delta$, the wave excites oscillations (phonons) of the trapped ions but then falls out of sync with those oscillations. After a period of time corresponding to half the amount of time needed for a gate operation, the same wave starts to decelerate the oscillation. Because the four electronic states corresponding to each of the four possible qubit combinations $(00,01,10$ or 11) couple to the lasers differently, the forces exerted on those states by the lasers are also different. The four states therefore move along different phase-generating pathways, so that the phase gain depends on the qubit combination. The phase information is then translated into qubit information by a simple operation that acts only on single qubits.

In previously reported two-qubit gates, ion displacement was performed adiabatically the time taken for a displacement that induces geometric phase changes was long compared to the period of oscillation of the ions, limiting the operating speed of the gates ${ }^{1,10}$. Schäfer et al. have overcome this speed limit by shaping the amplitude of the laser pulses precisely in time, so that phase change is generated from pathways of a different shape from those used previously. The adiabatic situation is akin to adjusting the working of a pendulum clock by gently wobbling the clock. Schäfer and colleagues' method is like hitting the pendulum repeatedly with well-timed hammer strikes.

Remarkably, the strikes are calibrated to work correctly no matter where in its oscillation the pendulum happens to be. This makes the logic-gate operation robust to fluctuations of and within the strikes - the fluctuations might change the pathways taken to generate geometric phase changes, but they leave the areas enclosed by the pathways unaffected. Using their method, the authors speed up their gates sufficiently to challenge the dogma that trapped-ion, two-qubit gates are slower than analogous solid-state systems, such as those that use superconducting or silicon-based qubits.

It remains to be seen whether trapped-ion qubits (or qubits based on other platforms, or combinations of qubit types) can be sufficiently well controlled and used in large enough numbers to implement a universal quantum computer. Even if all operations were to have fidelities of $99.9 \%$, a substantial number of additional qubits would still be required for quantum-error correction; these correction processes would take up additional computational time, slowing everything down. More experiments are needed in which gates are concatenated, to find out how this concatenation affects errors.

Schäfer et al. suggest that optimization of the parameters needed for the lasers, ion trapping and laser-qubit coupling will enable further speed increases and improve fidelities. However, classical computers will still be needed to control the protocols performed by quantum systems, and the speed limit for any qubit platform might be set by that classical computer. It should also be noted that all operations required for quantum computers will have different speed limits. Nevertheless, speeding up quantum logic gates, while at the same time mitigating or reducing the impact of most of the disturbances that affect them, is an excellent starting point for studying how the performance of quantum devices changes as their size increases, and potentially paves the way to a quantum computer.

Tobias Schaetz is at the Institute of Physics, Albert Ludwig University of Freiburg,

Freiburg 79104, Germany.

e-mail: tobias.schaetz@physik.uni-freiburg.de

1. Ballance, C. J., Harty, T. P., Linke, N. M., Sepiol, M. A. \& Lucas, D. M. Phys. Rev. Lett. 117, 060504 (2016).

2. Gaebler, J. P. et al. Phys. Rev. Lett. 117, 060505 (2016)

3. Cirac, J. I. \& Zoller, P. Phys. Rev. Lett. 74, 4091 (1995)

4. García-Ripoll, J. J., Zoller, P. \& Cirac, J. I. Phys. Rev. Lett. 91, 157901 (2003)

5. Duan, L.-M. Phys. Rev. Lett. 93, 100502 (2004).

6. García-Ripoll, J. J., Zoller, P. \& Cirac, J. I. Phys. Rev. A 71, 062309 (2005).

7. Steane, A. M., Imreh, G., Home, J. P. \& Leibfried, D. New J. Phys. 16, 053049 (2014).

8. Palmero, M., Martínez-Garaot, S., Leibfried, D., Wineland, D. J. \& Muga, J. G. Phys. Rev. A 95, 022328 (2017).

9. Schäfer, V. M. et al. Nature 555, 75-78 (2018).

10.Leibfried, D. et al. Nature 422, 412-415 (2003)

\title{
A gut feeling for cellular fate
}

\section{A population of progenitor cells in the midgut of fruit flies undergoes differentiation in response to mechanical force. This finding marks the first time that such a phenomenon has been reported in vivo. SEE LETTER P.103}

\section{JACKSON LIANG \& LUCY ERIN O'BRIEN}

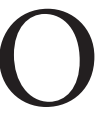
ver the past decade, advances in bioengineering have led to a newfound appreciation of the effects of mechanical force on stem cells. Micrometrescale culture systems that can subject cells to highly specific physical deformations have allowed researchers to demonstrate that force can modulate stem-cell behaviours, and even prime stem cells for therapeutic transplantation $^{1,2}$. However, even the most advanced culture systems merely approximate the complex and dynamic forces that stem cells experience in their native tissues. On page 103, He et al. ${ }^{3}$ combine sophisticated genetic approaches and innovative physical manipulations to investigate the role of force on stem cells in vivo. They 
make the striking discovery that mechanical force drives the differentiation of a specialized population of progenitor cells in the midgut of adult fruit flies (Drosophila melanogaster).

The fruit-fly midgut is equivalent to the stomach and small intestine of vertebrates. All digestive organs experience physical forces that are inherent in their physiological function: ingested food distends the gut, and muscle contractions compress it. These forces continuously deform the gut's epithelial lining, which includes both mature, differentiated cells (absorptive enterocytes and hormone-secreting enteroendocrine cells) and progenitor cells (stem cells and immature daughters that are committed to, but have not yet adopted, a particular differentiated identity).

He et al. identified a population of progenitor cells in the fly midgut that expresses the stretch-sensitive channel Piezo - a membrane-spanning, trimeric protein complex that opens in response to mechanical stimulation to allow the passage of ions across the membrane ${ }^{4}$. To trace these cells in vivo, the authors genetically engineered the Piezoexpressing cells such that they, and any cells that arose from them, produced a fluorescent protein. This analysis revealed that the Piezoexpressing cells mature into enteroendocrine cells. He and colleagues therefore named the population enteroendocrine precursors.

The fact that enteroendocrine precursors express Piezo suggested that they might respond to mechanical stimuli. The authors tested this possibility using two approaches. First, they distended the gut tube by feeding flies a diet containing indigestible methylcellulose. Second, they compressed gut tubes ex vivo using a microfluidic device. It has been established ${ }^{4}$ that mechano-activation of Piezo causes calcium ions $\left(\mathrm{Ca}^{2+}\right)$ to enter the cell's cytoplasm, and $\mathrm{He}$ et al. found that $\mathrm{Ca}^{2+}$ levels were significantly elevated in enteroendocrine precursors in both distended and compressed midguts. Crucially, $\mathrm{Ca}^{2+}$ levels were not elevated in distended or compressed midguts lacking the Piezo gene. These experiments convincingly demonstrated that the Piezo channel mediates $\mathrm{Ca}^{2+}$ influx in enteroendocrine precursors in response to mechanical stimuli (Fig. 1).

He et al. also observed that the midguts of Piezo-mutant flies failed to maintain normal numbers of enteroendocrine cells. This led the authors to hypothesize that Piezo-mediated $\mathrm{Ca}^{2+}$ influx might promote enteroendocrine differentiation. Consistent with this hypothesis, methylcellulose-distended midguts accumulated an excess of enteroendocrine cells. This effect required both Piezo and $\mathrm{Ca}^{2+}$ influx, and could be replicated in Piezo mutants by various genetic manipulations that increased cytoplasmic $\mathrm{Ca}^{2+}$ levels.

Taken together, these results support a scenario in which the activation of Piezo by mechanical force spurs enteroendocrine precursors to differentiate. Future investigation

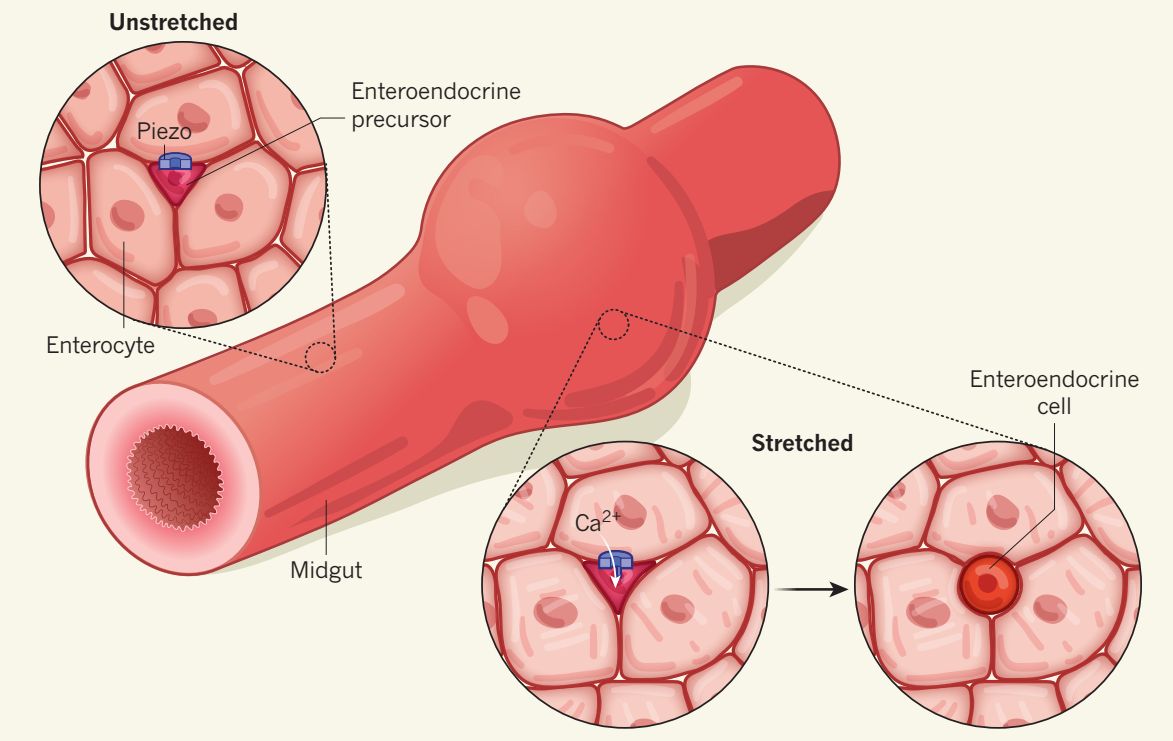

Figure 1 | Mechanosensing by specialized progenitor cells in vivo. The lining of the midgut of adult fruit flies contains differentiated intestinal cells, including enterocytes and enteroendocrine cells, and undifferentiated progenitor cells. He et al. ${ }^{3}$ report that a subset of progenitors called enteroendocrine precursors is characterized by expression of the ion-channel protein Piezo. In unstretched conditions, the channel is closed. However, the channel opens in response to mechanical forces that stretch cells - such as gut distension from taking a meal - to allow the influx of calcium ions $\left(\mathrm{Ca}^{2+}\right)$. This influx promotes precursor differentiation into hormone-secreting enteroendocrine cells.

should reveal the physiological purpose of mechanosensitive enteroendocrine-cell production. Until then, one possibility is that a larger population of enteroendocrine cells can more-efficiently produce the myriad hormones that coordinate local and systemic responses to ingested food.

How exactly do elevated $\mathrm{Ca}^{2+}$ levels promote enteroendocrine differentiation? Such differentiation requires limiting the activity of a membrane-spanning receptor protein called $\mathrm{Notch}^{5}$. He et al. found that elevated $\mathrm{Ca}^{2+}$ levels act to inhibit Notch in enteroendocrine precursors, thus permitting their differentiation.

Interestingly, this sensitivity of Notch to $\mathrm{Ca}^{2+}$ might be specific to enteroendocrine precursors - the authors found that levels of $\mathrm{Ca}^{2+}$ had no effect on Notch activation or differentiation in enterocyte precursor cells, and others have shown ${ }^{6}$ that the same is true of midgut stem cells. Instead, levels of $\mathrm{Ca}^{2+}$ in stem cells fluctuate in response to extrinsic inputs, such as nutrients, injury and stress, to control a switch between resting and proliferative states. These stark contrasts between enteroendocrine precursors, enterocyte precursors and stem cells raise intriguing questions about how different types of progenitor cell interpret the same chemical signal.

In addition, it seems that enteroendocrine differentiation is not the only cellular behaviour in the midgut to be affected by mechanical force. He et al. observed substantially more cell division in methylcellulose-distended midguts than in controls. However, they also found that enteroendocrine precursors rarely divide, at least under normal circumstances. One explanation that could reconcile these findings is that enteroendocrine precursors divide specifically in response to force. Another is that other, yet-unidentified, mechanosensitive progenitors exist in the midgut, and that they use a different mechanism to sense force and divide.

The identification of enteroendocrine precursors touches on a broader theme in stemcell biology: the existence of progenitors that are specialized for specific stimuli. Perhaps the best-understood example is injury-inducible 'reserve' stem cells, which are normally in a resting state but become activated by damage ${ }^{7}$. Now, He and colleagues add mechanical force to the list of stimuli that are associated with specialized progenitors. Many other stimuli might also populate this list. Indeed, recent studies $^{8-10}$ using single-cell RNA sequencing have found disarming diversity among mouse intestinal stem cells, at least at the level of gene expression. This diversity might hint that we have seen just the tip of the iceberg in terms of progenitor specialization.

Are there mechano-responsive progenitors in other organs? Three mammalian organs - the intestine, lungs and skeletal muscle would be attractive places to look. Like the fly midgut, these organs are both supported by progenitor cells and regularly subject to mechanical forces. A first step could simply be to examine them for progenitors that express Piezo or other mechanosensory channels.

Moving forward, a lack of microscale tools and protocols to manipulate force in adult tissues in vivo is likely to prove a bottleneck to progress. Such customized manipulations, which were crucial to the work of He and 
colleagues, are not easily translated between different organ systems. Perhaps the growing sophistication of three-dimensional culture systems and organ-on-a-chip devices will, over time, aid the development of technologies for mechanical manipulation of adult organs. More advances in this exciting area of research will no doubt reveal fundamental aspects of adult organ maintenance and improve strategies for tissue engineering.
Jackson Liang and Lucy Erin O'Brien are in the Department of Molecular and Cellular Physiology, Stanford University School of Medicine, Stanford, California 94305, USA. e-mail:lucye@stanford.edu

1. Kumar, A., Placone, J. K. \& Engler, A. J. Development 144, 4261-4270 (2017).

2. Vining, K. H. \& Mooney, D. J. Nature Rev. Mol. Cell Biol. 18, 728-742 (2017).

3. He, L., Si, G., Huang, J., Samuel, A. D. T. \&
Perrimon, N. Nature 555, 103-106 (2018)

4. Wu, J., Lewis, A. H. \& Grandl, J. Trends Biochem. Sci. 42, 57-71 (2017)

5. Sallé, J. et al. EMBO J. 36, 1928-1945 (2017).

6. Deng, H., Gerencser, A. A. \& Jasper, H. Nature $\mathbf{5 2 8}$ 212-217 (2015).

7. Li, L. \& Clevers, H. Science 327, 542-545 (2010).

8. Yan, K. S. et al. Cell Stem Cell 21, 78-90 (2017).

9. Haber, A. L. et al. Nature 551, 333-339 (2017).

10. Barriga, F. M. et al. Cell Stem Cell 20, 801-816 (2017)

This article was published online on 7 February 2018.

\section{Quantum}

\section{upside-down cake}

\section{Exotic states of matter called topological superconductors have potential applications in quantum computing, but have been difficult to produce in more than one dimension. A way of overcoming this limitation has now been found.}

\section{CHIH-KANG SHIH \& ALLAN H. MACDONALD}

$\mathrm{O}$ ver the past decade, there has been explosive growth in the study of condensed-matter systems that have striking properties associated with unusual topologies ${ }^{1}$. The new kid on the block, experimentally speaking, is the topological superconductor. Like all superconductors, a topological one can transfer electric current without energy dissipation. But it also displays exotic excitations called Majorana modes, which are being investigated for use in quantum computing because they are extremely resistant to external interference ${ }^{2}$. Onedimensional topological superconductors have previously been reported $^{3,4}$. Writing in Nature Communications, Ménard et al. ${ }^{5}$ describe a technique for generating such superconductors in two dimensions.

Topological superconductors (TSCs) can be produced by combining three ingredients: ordinary superconductivity; strong coupling between the spins (magnetic moments) of electrons and their orbital motion; and the breaking of a fundamental symmetry of nature called time-reversal symmetry. The first-reported 1D TSC was realized using an ordinary superconductor, a nanoscale semiconducting wire that had strong spin-orbit coupling, and applied external magnetic fields ${ }^{3}$. Another 1D TSC was subsequently generated by placing ordered chains of magnetic atoms on the surface of superconducting lead, which has strong spin-orbit coupling ${ }^{4}$. In

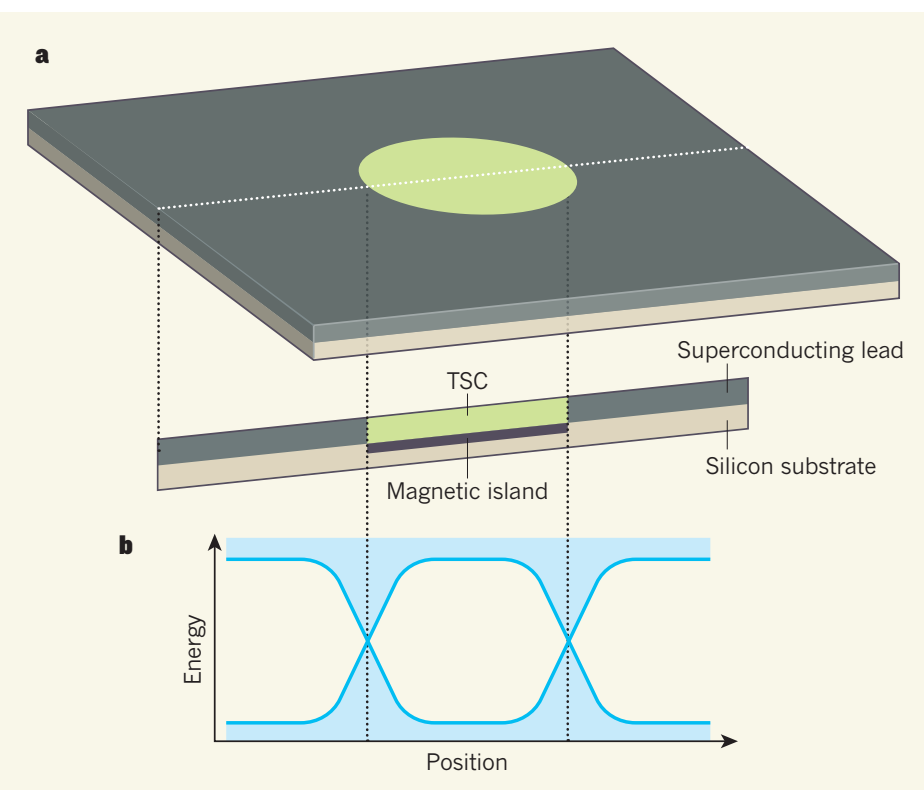

Figure 1 | Generation of a 2D topological superconductor. Ménard et al. ${ }^{5}$ report a technique for producing exotic states of matter called topological superconductors (TSCs) in two dimensions. Such materials exhibit superconductivity, whereby electric current can be transferred without energy dissipation, and show excitations called Majorana edge modes, which could be useful in quantum computing. a, Ménard and colleagues' experiment consists of a silicon substrate, structures called magnetic islands (for simplicity, a single island is shown here) and an atomically thin layer of superconducting lead. The authors report the generation of a TSC in the region of the lead directly above a magnetic island. b, Ménard et al. obtain an energy spectrum for their system that shows the possible energies that electrons can have (light blue) at different positions. They detect X-shaped features in the spectrum at positions corresponding to magneticisland boundaries that they interpret as evidence of Majorana edge modes. both cases, signatures of localized Majorana modes were observed at the ends of the TSC.

Unlike these 1D examples, a 2D TSC supports Majorana modes that are free to propagate ${ }^{1}$, a potentially desirable feature for quantum-computing applications. Such modes travel along the edge of the TSC and are chiral, meaning that they move in just one direction. This chiral property produces a characterismomentum space - a theoretical space that is mathematically equivalent to real space, which offers an alternative perspective of quantum systems by replacing positions with momenta. One method for producing a 2D TSC is to couple the excitations that exist on the surface of a 3D topological insulator to an ordinary $2 \mathrm{D}$ superconductor ${ }^{6,7}$. An alternative approach is to link a $2 \mathrm{D}$ superconductor that has strong spin-orbit coupling to a $2 \mathrm{D}$ magnetic system ${ }^{8}$.

Ménard and colleagues' work is probably the first successful realization of a 2D TSC based on the latter approach. The authors produced a $2 \mathrm{D}$ magneticsuperconductor composite system using a method known as epitaxy, whereby materials are grown on top of a substrate layer by layer. In this method, magnetic materials naturally separate into an array of isolated patches known as islands. The authors' experimental set-up consisted of a silicon substrate, an atomically thin film of superconducting lead, and magnetic islands made of cobalt.

Although ultrathin lead films are routinely grown on silicon substrates with atomic precision $^{9}$, the growth of magnetic islands on top of such films is technically challenging because the structural integrity of the film is often disrupted. Ménard et al. turned this strategy upside down: they grew their composite system in such a way that the magnetic islands formed underneath the superconducting-lead film (Fig. 1a). 